\title{
Urinary Neutrophil Gelatinase-Associated Lipocalin Is a Potential Biomarker for Renal Damage in Patients with Systemic Lupus Erythematosus
}

\author{
Chun-Chen Yang,, Song-Chou Hsieh, ${ }^{2}$ Ko-Jen Li, ${ }^{2}$ Cheng-Han Wu, ${ }^{2}$ \\ Ming-Chi Lu, ${ }^{3}$ Chang-Youh Tsai, ${ }^{4}$ and Chia-Li Yu, ${ }^{1,2}$ \\ ${ }^{1}$ Department of Internal Medicine, Keelung City Municipal Hospital, Keelung 201, Taiwan \\ ${ }^{2}$ Department of Internal Medicine, National Taiwan University Hospital and National Taiwan University College of Medicine, \\ No. 7 Chung-Shan South Road, Taipei 100, Taiwan \\ ${ }^{3}$ Division of Immunology, Rheumatology and Allergy, Buddhist Dalin Tzu-Chi General Hospital, Dalin, Chiayi 622, Taiwan \\ ${ }^{4}$ Section of Allergy, Immunology and Rheumatology, Taipei Veterans General Hospital, Taipei 112, Taiwan
}

Correspondence should be addressed to Chia-Li Yu, chialiyu@ntu.edu.tw

Received 1 July 2011; Accepted 8 November 2011

Academic Editor: Brian Poole

Copyright () 2012 Chun-Chen Yang et al. This is an open access article distributed under the Creative Commons Attribution License, which permits unrestricted use, distribution, and reproduction in any medium, provided the original work is properly cited.

\begin{abstract}
Neutrophil gelatinase-associated lipocalin (NGAL) has been demonstrated to be a novel biomarker in acute and chronic kidney disease. We hypothesized that 24-hour urinary NGAL excretion may be a predictor for renal damage in patients with systemic lupus erythematosus (SLE). Thirty-four SLE patients with renal involvement (SLE-renal group), 8 SLE patients without renal involvement (SLE-nonrenal group), 14 patients with non-SLE autoimmune diseases (disease control or DC group), and 12 healthy volunteers (normal control or NC group) were compared for 24-hour urinary excretion of NGAL and different cytokines. We found that the 24-hour urinary NGAL excretion in the SLE-renal group was higher than that in the SLE-non-renal, DC, and NC groups. However, the excretion of interleukin-10, transforming growth factor- $\beta 1$, and tumor necrosis factor- $\alpha$ was not different between the SLE-renal and SLE-non-renal groups. Furthermore, NGAL excretion in the SLE-renal group was correlated with serum creatinine levels and creatinine clearance, but not with the SLE Disease Activity Index score. Multivariate logistic regression analysis and receiver operating characteristic curve analysis revealed that 24-hour urinary NGAL excretion is a potential biomarker for renal damage in SLE patients, with higher sensitivity and specificity than anti-dsDNA antibody titers.
\end{abstract}

\section{Introduction}

Systemic lupus erythematosus (SLE) is a chronic inflammatory autoimmune disease affecting many organ systems. Lupus nephritis (LN) is a common and serious complication involving a major organ in patients with SLE. From one-third to one-half of SLE patients have different degrees of renal damage [1] ranging from asymptomatic hematuria/proteinuria to overt nephritis, rapidly progressive glomerulonephritis, nephrotic syndrome, or renal failure [2].

Recent advances in the diagnosis and treatment of LN have resulted in markedly improved renal function and survival in SLE patients with renal survival rates of $83-92 \%$ and $74-84 \%$ after 5 and 10 years, respectively [3]. However, the incidence of LN flareup in different studies is variable from $27-66 \%$ [4], rendering LN, a major cause of morbidity and mortality in SLE patients. Since $10-26 \%$ patients with LN progress to end-stage renal disease [5], early detection and effective treatment of LN is important for the prevention of renal failure or mortality in SLE patients.

At present, renal biopsy remains the gold standard in establishing the diagnosis and prognosis of LN that can guide treatment decisions. However, renal biopsy is not routinely performed serially and does not reliably represent the global status of the kidney [1]. In contrast, other noninvasive procedures for monitoring LN include the measurement 
of serum creatinine levels, 24-hour creatinine clearance (Ccr), 24-hour urine protein amounts, antidouble stranded DNA (anti-dsDNA) antibody titers, levels of complements 3 (C3) and C4, and the presence of urine sediments $[2,6]$. Nevertheless, the diagnostic value of these measurements for LN remains controversial.

Urine is an ideal source of potential biomarkers for LN, because of its easy accessibility and the fact that it can directly reflect the status of local inflammation/damage in the kidney. In recent years, many studies have proposed different cytokines/chemokines such as transforming growth factor (TGF)- $\beta$ [7-9], interferon (IFN)- $\gamma$, interleukin (IL)-2 [10], monocyte chemoattractant protein-1/chemokine ligand 2 (MCP-1/CCL2) [7, 11], IFN- $\gamma$ inducible protein-10/CXC chemokine ligand-10 (IP-10/CXCL10), CXC chemokine receptor 3 (CXCR3) [8], and tumor necrosis factor (TNF) like weak inducer of apoptosis (TWEAK) $[12,13]$ as well as adhesion molecules including vascular cell adhesive molecule-1 (VCAM-1) and P-selectin [14] as useful biomarkers for LN [6]. However, none of these molecules have been validated to date in clinical applications.

Neutrophil gelatinase-associated lipocalin (NGAL), a $25-\mathrm{kDa}$ small protein belonging to the lipocalin protein superfamily, is specialized in binding and transporting small hydrophobic molecules including iron [15]. NGAL has been recently demonstrated to be an early biomarker in acute kidney injury after cardiopulmonary bypass, major cardiac surgery, elective cardiac catheterization and angiography, hemolytic uremic syndrome, and kidney transplantation. NGAL is also a candidate biomarker for chronic kidney diseases, such as immunoglobulin A (IgA) nephropathy, membranous and membranoproliferative glomerulonephritis, autosomal dominant polycystic kidney disease, and pediatric LN $[16,17]$. Based on these findings, we hypothesized that urinary NGAL excretion may be a potential biomarker for renal damage/inflammation in LN.

In the present study, we measured 24-hour urinary NGAL excretion using a commercially available enzymelinked immunosorbent assay (ELISA) kit in SLE patients with and without renal involvement and compared this to the excretion of the cytokines IL-10, TGF- $\beta 1$, and TNF- $\alpha$. In addition, to determine the specificity of NGAL for LN, we also measured 24-hour urinary NGAL excretion in non-SLE patients with other autoimmune diseases. Our results suggest that urinary NGAL excretion is indeed a potential biomarker for renal damage in patients with SLE.

\section{Materials and Methods}

2.1. Patients and Controls. Forty-two patients fulfilling the 1997 revised American College of Rheumatology classification criteria for SLE [18] were recruited. These patients were further divided into SLE-active nephritis (active LN, $n=24$ ), SLE-renal (active LN, $n=24$; and proteinuria alone, $>0.5 \mathrm{~g} /$ day, $n=10)$, and SLE-non-renal $(n=8)$ groups. The SLE-active nephritis group was defined as the presence of any of the following abnormal parameters in the urinalysis: hematuria ( $>5$ red blood cells/high power field
(HPF); exclusion of stones, infection, or other causes), pyuria ( $>5$ leukocytes/HPF; exclusion of infection), urinary casts (granular or red blood cell casts); a $>30 \%$ increase in serum creatinine levels within 3 months; or biopsy-proven nephritis. The SLE-renal group included all patients from the SLEactive nephritis group as well as SLE patients with proteinuria ( $>0.5 \mathrm{~g} /$ day) alone, without abnormal urine sediments. The SLE-nonrenal group consisted of SLE patients without abnormal urinalysis results or renal function impairment on the day of the study. The disease control (DC) group consisted of 14 patients with non-SLE autoimmune diseases. Twelve healthy volunteers served as the normal control (NC) group. Subjects with current infection (especially urinary tract infection) and diabetes mellitus were excluded. This study was approved by the local IRB and Ethical Committee, National Taiwan University Hospital, Taipei, Taiwan. Written informed consent was obtained from all participants.

2.2. Data Collection. Medical records of all patients were reviewed. The demographic, laboratory, and clinical data were collected and are summarized in Table 1.

2.3. Assessment of Disease Activity. Disease activity of the SLE patients was assessed by the SLE Disease Activity Index-2000 (SLEDAI-2 K) [19] on the day of urine collection.

\subsection{Measurement of Urinary NGAL and Different Cytokines} by ELISA. The 24-hour urine amount was measured after thorough mixing. Ten $\mathrm{mL}$ of each urine sample was then centrifuged within 2 hours after collection at 3,000 rpm for 10 minutes to remove cellular debris. The cleaned urine samples were stored at $-80^{\circ} \mathrm{C}$ until measurement. The concentration of NGAL, IL-10, TGF- $\beta 1$, and TNF- $\alpha$ was quantified by commercial NGAL (Quantikine; R\&D Systems, Inc.), IL-10 (DuoSet; R\&D Systems, Inc.), TGF- $\beta 1$ (Quantikine; R\&D Systems, Inc.), and TNF- $\alpha$ (OptEIA Set; BD Biosciences Pharmingen) ELISA kits, in accordance with the respective manufacturer's instructions. The TGF- $\beta 1$ ELISA kit detected the active form of TGF- $\beta 1$ by treating urine samples with acid to activate TGF- $\beta 1$ immediately before measurement. The values of 24-hour urinary excretion of NGAL, IL-10, TGF- $\beta 1$, and TNF- $\alpha$ were calculated by multiplying the urine concentration by the urine amount.

2.5. Statistical Analysis. Statistical analysis was conducted using Statistical Package for Social Sciences version 13.0 for Window software (SPSS Inc.). Because the data of continuous variables were mostly not normally distributed, they were presented in terms of median with interquartile range (IQR), which represents central tendency and spread. For continuous variables, testing between 2 groups was performed by the Mann-Whitney $U$ test. Categorical variables were compared by Pearson's Chi-Square test or by Fisher's exact test when very small proportions were analyzed. Correlations among continuous variables were calculated by the Spearman rank correlation coefficient $\left(r_{s}\right)$. Logistic regression analysis was performed to determine predictors for renal damage in SLE patients. To determine the 
TABLE 1: Demographic, laboratory, and clinical characteristics of the different patient groups. ${ }^{\mathrm{a}} \mathrm{b}$

\begin{tabular}{|c|c|c|c|c|}
\hline \multirow{2}{*}{ Clinical parameter } & \multicolumn{3}{|c|}{ SLE } & \multirow{2}{*}{ DC } \\
\hline & $\begin{array}{l}\text { SLE-active } \\
\text { nephritis }\end{array}$ & SLE-renal & SLE-non-renal & \\
\hline Patients (number) & 24 & 34 & 8 & 14 \\
\hline Age (years) & $33(16)(24)$ & $33(12)(34)$ & $36(7)(8)$ & $36(26)(14)$ \\
\hline Female: male ratio & $18: 6(24)$ & $27: 7(34)$ & $8: 0(8)$ & $14: 0(14)$ \\
\hline \multicolumn{5}{|l|}{ Laboratory data } \\
\hline Leukocyte $\left(10^{9} / \mathrm{I}\right)$ & $7.26(4.92)(24)$ & $6.47(5.47)(34)$ & $6.73(5.80)(8)$ & $9.33(9.28)(13)$ \\
\hline Neutrophil $\left(10^{9} / \mathrm{I}\right)$ & $5.27(4.62)(16)$ & $4.27(5.52)(23)$ & $4.48(5.82)(5)$ & $7.45(6.62)(6)$ \\
\hline Lymphocyte $\left(10^{9} / \mathrm{I}\right)^{\dagger, \S \S}$ & $0.76(0.71)(16)$ & $0.64(0.65)(23)$ & $1.45(1.30)(5)$ & $2.12(1.65)(6)$ \\
\hline Hemoglobin $(g / I)^{\dagger \dagger, \S \S}$ & $95(24)(24)$ & $99(24)(34)$ & $115(33)(8)$ & $117(18)(13)$ \\
\hline Platelet count $\left(10^{9} / \mathrm{I}\right)$ & $210(180)(24)$ & $199(145)(34)$ & $252(334)(8)$ & $198(159)(13)$ \\
\hline Serum creatinine $(\mu \mathrm{moI} / \mathrm{I})^{\dagger+, \S \S}$ & $124(283)(24)$ & $106(160)(34)$ & $71(9)(8)$ & $62(88)(14)$ \\
\hline $\operatorname{Serum} \operatorname{albumin}(\mathrm{g} / \mathrm{I})^{* *, \dagger \dagger, \&, \S \S}$ & $28(8)(23)$ & $30(8)(31)$ & $43(3)(7)$ & $41(7)(12)$ \\
\hline 24-hour Ccr (mL/sec) & $0.72(1.32)(20)$ & $0.89(1.29)(24)$ & $1.09(-)(3)$ & $4.91(0.27)(7)$ \\
\hline 24-hour urine protein $(\mathrm{g} / \text { day })^{* *, \dagger+, \&, \S \S}$ & $2.82(7.59)(23)$ & $2.26(4.64)(33)$ & $0.06(0.05)(8)$ & $0.04(0.11)(13)$ \\
\hline ESR 1 hour (mm) & $44(32)(16)$ & $49(30)(22)$ & $31(46)(5)$ & $30(46)(10)$ \\
\hline $\mathrm{C} 3(\mathrm{~g} / \mathrm{I})^{*, \dagger+, \#, \S \S}$ & $0.56(0.47)(23)$ & $0.60(0.54)(31)$ & $0.80(0.37)(8)$ & $1.24(0.41)(12)$ \\
\hline $\mathrm{C} 4(\mathrm{~g} / \mathrm{I})$ & $0.13(0.16)(18)$ & $0.14(0.15)(26)$ & $0.13(0.13)(8)$ & $0.19(0.10)(13)$ \\
\hline Positive anti-dsDNA ${ }^{t, \S}$ & $12 / 24(50)$ & $15 / 32(46.9)$ & $3 / 8(37.5)$ & $0 / 7(0)$ \\
\hline \multicolumn{5}{|l|}{ Disease activity } \\
\hline Total SLEDAI**,\& & $15(10)(23)$ & $14(10)(29)$ & $8(5)(8)$ & - \\
\hline Extrarenal SLEDAI & $4(4)(23)$ & $4(7)(29)$ & $8(5)(8)$ & - \\
\hline Renal SLEDAI $* *, \&$ & $12(4)(23)$ & $8(8)(32)$ & $0(0)(8)$ & - \\
\hline Proteinuria $>0.5 \mathrm{~g} /$ day $^{* *, \&}$ & $19 / 23(82.6)$ & $29 / 33(87.9)$ & $0 / 8(0)$ & - \\
\hline Hematuria $^{* *, \&}$ & $20 / 24(83.3)$ & $20 / 33(60.6)$ & $0 / 8(0)$ & - \\
\hline Pyuria* & $10 / 24(41.7)$ & $10 / 33(30.3)$ & $0 / 8(0)$ & - \\
\hline Urinary casts & $9 / 24(37.5)$ & $9 / 33(27.3)$ & $0 / 8(0)$ & - \\
\hline \multicolumn{5}{|l|}{ Clinical features } \\
\hline Active nephritis & $24 / 24(100)$ & $24 / 34(70.6)$ & $0 / 8(0)$ & $0 / 14(0)$ \\
\hline Leukopenia & $1 / 24(4.2)$ & $5 / 34(14.7)$ & $1 / 8(12.5)$ & $0 / 13(0)$ \\
\hline Lymphopenia $^{\dagger, \S \S}$ & $13 / 16(81.3)$ & $19 / 23(82.6)$ & $3 / 5(60)$ & $1 / 6(16.7)$ \\
\hline Thrombocytopenia & $2 / 24(8.3)$ & $3 / 34(8.8)$ & $0 / 8(0)$ & $0 / 13(0)$ \\
\hline Low complement ${ }^{\dagger, \S}$ & $20 / 24(83.3)$ & $27 / 33(81.8)$ & $5 / 8(62.5)$ & $6 / 14(42.9)$ \\
\hline Arthritis & $1 / 24(4.2)$ & $2 / 34(5.9)$ & $0 / 8(0)$ & - \\
\hline Rash & $5 / 24(20.8)$ & $8 / 34(23.5)$ & $2 / 8(25)$ & - \\
\hline Serositis & $2 / 24(8.3)$ & $2 / 34(5.9)$ & $0 / 8(0)$ & - \\
\hline CNS involvement & $3 / 24(12.5)$ & $5 / 34(14.7)$ & $2 / 8(25)$ & - \\
\hline Vasculitis & $1 / 24(4.2)$ & $3 / 34(8.8)$ & $2 / 8(25)$ & - \\
\hline Disease duration (years) & $3.5(7.5)(24)$ & $5.0(9.5)(34)$ & $3.1(3.8)(8)$ & - \\
\hline \multicolumn{5}{|l|}{ Drug treatment } \\
\hline Steroids ${ }^{\dagger \dagger, \S \S}$ & $24 / 24(100)$ & $34 / 34(100)$ & $8 / 8(100)$ & 9/14 (64.3) \\
\hline $\begin{array}{l}\text { Daily prednisolone or equivalent dosage } \\
(\mathrm{mg} / \text { day })^{\mathrm{ct}, \S}\end{array}$ & $18.4(17)(24)$ & $18(14)(34)$ & $13(20)(8)$ & $7(20)(13)$ \\
\hline Hydroxychloroquine & $22 / 24(91.7)$ & $30 / 34(88.2)$ & $8 / 8(100)$ & $12 / 14(85.7)$ \\
\hline Azathioprine & $6 / 24(25)$ & $11 / 34(32.4)$ & $0 / 8(0)$ & $3 / 14(21.4)$ \\
\hline Mycophenolate mofetil & $1 / 24(4.2)$ & $2 / 34(5.9)$ & $0 / 8(0)$ & $0 / 14(0)$ \\
\hline
\end{tabular}


TABle 1: Continued.

\begin{tabular}{|c|c|c|c|c|}
\hline \multirow{2}{*}{ Clinical parameter } & \multicolumn{3}{|c|}{ SLE } & \multirow{2}{*}{ DC } \\
\hline & $\begin{array}{l}\text { SLE-active } \\
\text { nephritis }\end{array}$ & SLE-renal & SLE-non-renal & \\
\hline Cyclophosphamide & 4/24 (16.7) & $4 / 34(11.8)$ & $2 / 8(25)$ & $1 / 14(7.1)$ \\
\hline Aspirin or NSAIDs & $9 / 24(37.5)$ & $14 / 34(41.2)$ & $6 / 8(75)$ & $7 / 14(50)$ \\
\hline ACEIs or ARBs $*, \uparrow+, \S$ & $10 / 24(41.7)$ & $12 / 34(35.3)$ & $0 / 8(0)$ & $0 / 14(0)$ \\
\hline Pulse therapy ${ }^{d}$ & $11 / 24(45.8)$ & $12 / 34(35.3)$ & $3 / 8(37.5)$ & $3 / 14(21.4)$ \\
\hline
\end{tabular}

${ }^{a}$ In continuous variables, data are shown as median (IQR) (number). In categorical variables, data are shown as number (percent).

$\mathrm{b} * P<0.05$ and ${ }^{* *} P<0.01$ for SLE-active nephritis group versus SLE non-renal group; ${ }^{\dagger} P<0.05$ and ${ }^{\dagger \dagger} P<0.01$ for SLE-active nephritis group versus DC group; ${ }^{\#} P<0.01$ for SLE-non-renal group versus DC group; ${ }^{* \&}<0.01$ for SLE-renal group versus SLE-non-renal group; ${ }^{\S} P<0.05$ and $\$ \S P<0.01$ for SLErenal group versus DC group.

${ }^{\mathrm{c}}$ The mean daily dosage of prednisolone or its equivalent during the preceding 30 days.

${ }^{\mathrm{d}}$ Numbers of patients who were treated by methylprednisolone or cyclophosphamide pulse therapy during the preceding 30 days.

SLE: systemic lupus erythematosus; DC: disease control; Ccr: creatinine clearance; ESR: erythrocyte sedimentation rate; C3: complement 3; C4: complement 4; anti-dsDNA: anti-double stranded DNA; SLEDAI: SLE Disease Activity Index; CNS: central nervous system; NSAIDs: nonsteroidal anti-inflammatory drugs; ACEIs: angiotensin-converting enzyme inhibitors; ARBs: angiotensin II receptor antagonists; IQR: interquartile range.

TABLE 2: 24-hour urinary excretion of NGAL, IL-10, TGF- $\beta 1$, and TNF- $\alpha$ in the study groups. ${ }^{\text {a }}$

\begin{tabular}{|c|c|c|c|c|c|}
\hline \multirow[b]{3}{*}{ Number } & \multicolumn{3}{|c|}{ SLE patients } & \multicolumn{2}{|c|}{ Controls } \\
\hline & Active nephritis & Renal & Non-renal & DC & $\mathrm{NC}$ \\
\hline & 24 & 34 & 8 & 14 & 12 \\
\hline NGAL (mg/day) & $50.41(199.93)$ & $49.05(114.45)$ & $21.78(12.42)$ & $13.94(12.53)$ & $7.65(7.64)$ \\
\hline IL-10 (ng/day) & $2.34(16.81)$ & $3.45(21.06)$ & $10.03(18.27)$ & $13.12(15.99)$ & $5.40(24.09)$ \\
\hline TGF- $\beta 1$ (ng/day) & $41.54(75.59)$ & $23.82(69.08)$ & $16.17(16.13)$ & $15.57(14.94)$ & $7.83(6.39)$ \\
\hline TNF- $\alpha$ (ng/day) & $6.01(15.23)$ & $5.85(17.63)$ & $20.49(24.26)$ & $26.68(39.04)$ & $0(13.65)$ \\
\hline
\end{tabular}

${ }^{a}$ Data are shown as median (IQR).

NGAL: neutrophil gelatinase-associated lipocalin; IL-10: interleukin-10; TGF- $\beta 1$ : transforming growth factor- $\beta 1$; TNF- $\alpha$ : tumor necrosis factor- $\alpha$; SLE: systemic lupus erythematosus; DC: disease control; NC: normal control; IQR: interquartile range.

sensitivity, specificity, positive predictive values, and negative predictive values of 24-hour urinary NGAL excretion at different cutoff values for the diagnosis of renal involvement in SLE patients, a conventional receiver operating characteristic (ROC) curve was generated. An area under the curve (AUC) of 0.5 was considered no better than expected by chance, whereas a value of 1.0 signified a perfect biomarker. Twotailed $P$ values of less than 0.05 were considered statistically significant. For graphing purposes, the highly skewed 24hour urinary excretion data of NGAL, IL-10, and TGF- $\beta 1$ were log-transformed, in which case a constant of 1.00 was added to each value of urinary NGAL, IL-10, and TGF- $\beta 1$ excretion in order to move the minimum value to 1.00 .

\section{Results}

3.1. Demographic and Clinical Characteristics of the Study Groups. The present study comprised 42 SLE patients (ratio of females to males, 35:7; median age, 34 years; IQR, 11) including 24 SLE patients with active LN, 10 SLE patients with proteinuria only, and 8 SLE patients without renal involvement. In addition, the study included 14 patients without SLE but with other autoimmune diseases (4 had Sjogren's syndrome; 3 , anti-phospholipid syndrome; 1 , vasculitis; 1, autoimmune hemolytic anemia; 1, rheumatoid arthritis with systemic sclerosis overlap; 1, polymyositis; 1, adult-onset Still's disease; 1, pemphigus; 1, acute urticaria) as the DC group (ratio of females to males, 14:0; median age, 36 years; IQR, 26) and 12 healthy volunteers as the NC group (ratio of females to males, $10: 2$; median age, 32 years; IQR, 12). There was no significant difference in age or female-male ratio among SLE, DC, and NC groups. The demographic, laboratory, and clinical characteristics of the different patient groups are summarized in Table 1. These data reveal that the SLE-renal group had higher SLE Disease Activity Index (SLEDAI) scores (median, 14; IQR, 10 versus median, 8; IQR, $5 ; P=0.000)$ than did the SLE-non-renal group.

3.2. 24-Hour Urinary Excretion of NGAL, IL-10, TGF- $\beta 1$, and $T N F-\alpha$. Urinary NGAL excretion in the SLE-active nephritis and SLE-renal groups was significantly higher than that in the SLE-non-renal, DC, and NC groups (Figure 1(a) and Table 2). These results suggest that urinary NGAL may be a potential biomarker for SLE patients with renal inflammation or damage. Unexpectedly, no significant difference in urinary IL-10 excretion was observed among these groups (Figure 1(b)). Urinary TGF- $\beta 1$ excretion was found to be higher in the 3 SLE subgroups and in the DC group than in the NC group (Figure 1(c)); however, no significant difference was found among the 3 SLE subgroups. Interestingly, urinary TNF- $\alpha$ excretion in the SLE-active nephritis group was not different from that of the NC group. 


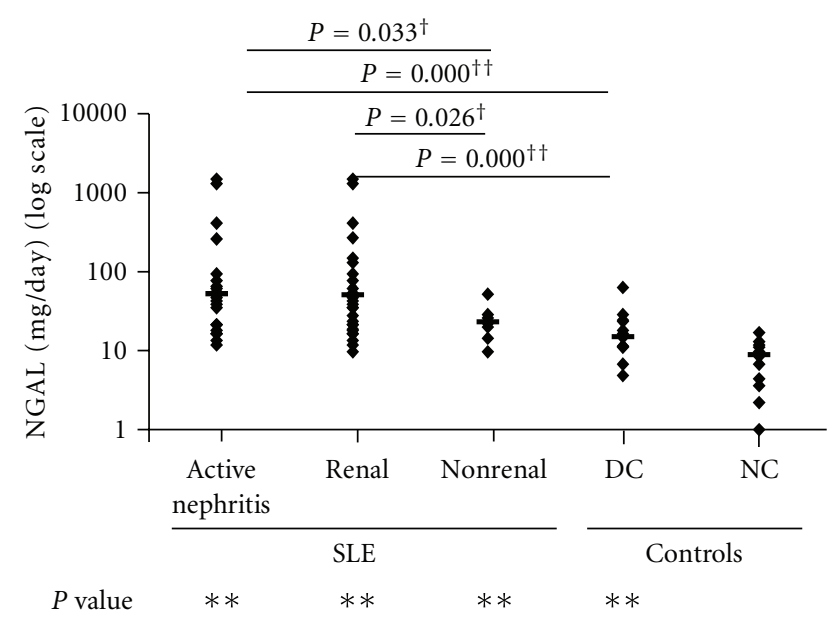

(a)

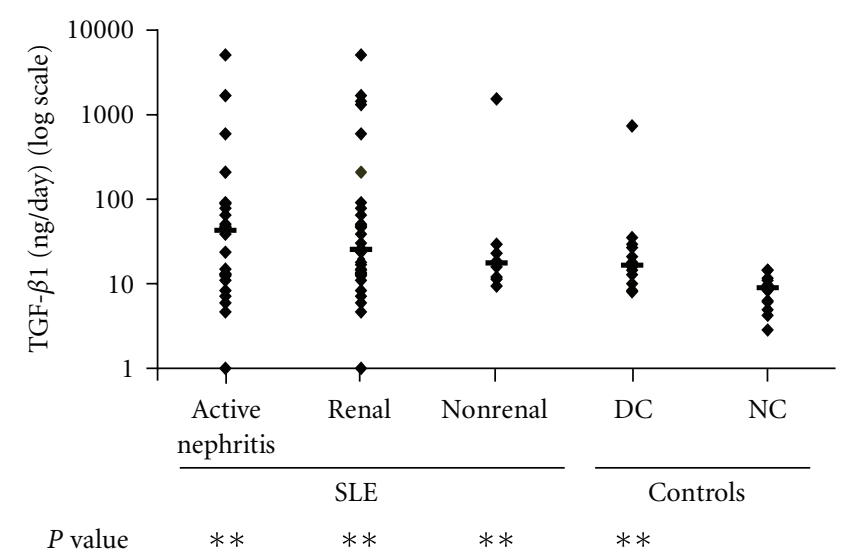

(c)

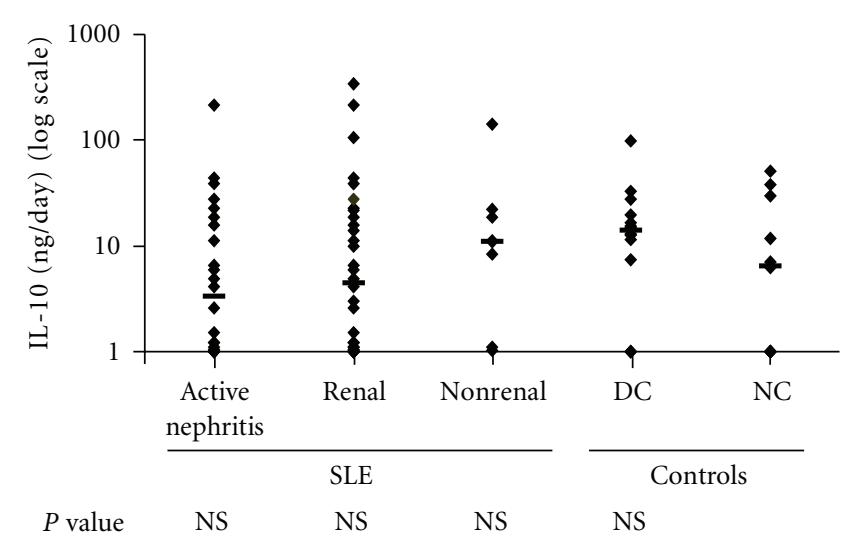

(b)

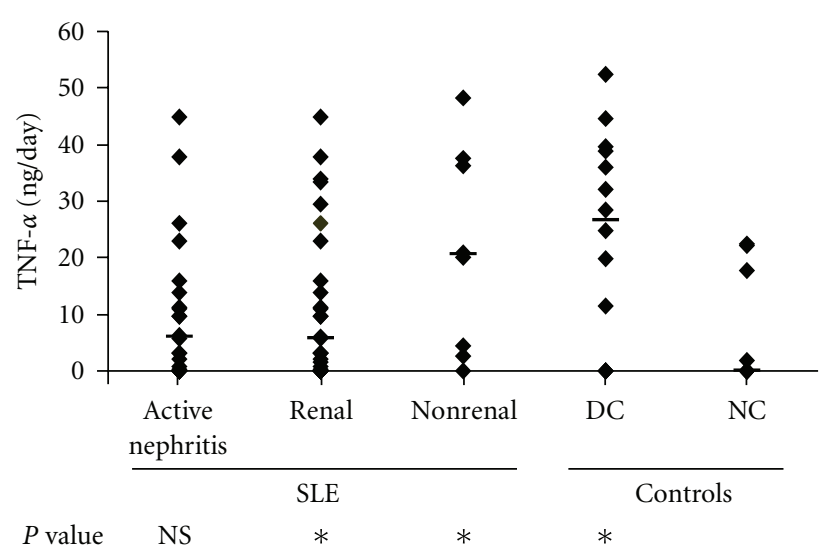

(d)

FIgURE 1: Comparison of 24-hour urinary excretion of neutrophil gelatinase-associated lipocalin (NGAL) (a), interleukin (IL)-10 (b), transforming growth factor (TGF)- $\beta 1$ (c), and tumor necrosis factor (TNF)- $\alpha$ (d) in 5 groups: systemic lupus erythematosus (SLE)-active nephritis $(n=24)$, SLE-renal (24 SLE-active nephritis + 10 SLE-proteinuria only, $n=34)$, SLE-non-renal $(n=8)$, disease control (DC, $n=14$ ), and normal control (NC, $n=12$ ) groups. ${ }^{\dagger} P<0.05$ and ${ }^{\dagger \dagger} P<0.01$, are shown above panels, for comparison among the different patient groups. ${ }^{*} P<0.05,{ }^{* *} P<0.01$, and NS, not significant, are shown below the $x$-axis of panels, in comparison with the NC group. The median value in each study group is shown by a horizontal line.

However, urinary TNF- $\alpha$ excretion was higher in the SLErenal, SLE-non-renal, and DC groups than in the NC group (Figure 1(d)). On the other hand, there was no significant difference in urinary TNF- $\alpha$ excretion among the 3 SLE subgroups. These results may suggest that urinary excretion of TGF- $\beta 1$ and TNF- $\alpha$, but not IL-10, was higher in patients with SLE and other autoimmune diseases, whereas urinary NGAL excretion was higher only in SLE patients with renal involvement.

3.3. Correlations among 24-Hour Urinary Excretion of NGAL, IL-10, TGF- $\beta 1$, and TNF- $\alpha$ in the SLE-Renal Group. Although urinary NGAL excretion was higher in the SLErenal group, it was not correlated with the excretion of IL-10 (Figure 2(a)), TGF- $\beta 1$ (Figure 2(c)), or TNF- $\alpha$ (Figure 2(e)). These results indicate that NGAL is probably a biomarker for renal damage, rather than for immune-mediated kidney inflammation as reported by other authors $[16,17]$. On the other hand, a negative correlation was found between IL-10 and TGF- $\beta 1\left(r_{s}=-0.506, P=0.001\right.$; Figure 2(b)), whereas a tendency towards positive correlation between IL10 and TNF- $\alpha\left(r_{s}=0.297, P=0.056\right.$; Figure $\left.2(\mathrm{~d})\right)$ and a tendency towards negative correlation between TGF- $\beta 1$ and TNF- $\alpha\left(r_{s}=-0.273, P=0.080\right.$; Figure 2(f) $)$ were observed. These results indicate the presence of an intricate regulatory network that includes the processes of inflammation/antiinflammation, tissue repair/tissue fibrosis, and cell proliferation/differentiation in renal damage sites in SLE patients.

3.4. Correlations of 24-Hour Urinary Excretion of NGAL with Renal Functional Parameters and Disease Activity in the SLE-Renal Group. In SLE patients with renal involvement, urinary NGAL excretion was positively correlated with serum creatinine levels $\left(r_{s}=0.394, P=0.010\right.$; Figure $\left.3(\mathrm{a})\right)$, negatively correlated with 24-hour Ccr $\left(r_{s}=-0.435, P=\right.$ 0.023 ; Figure $3(\mathrm{~b})$ ) and not correlated with serum albumin 


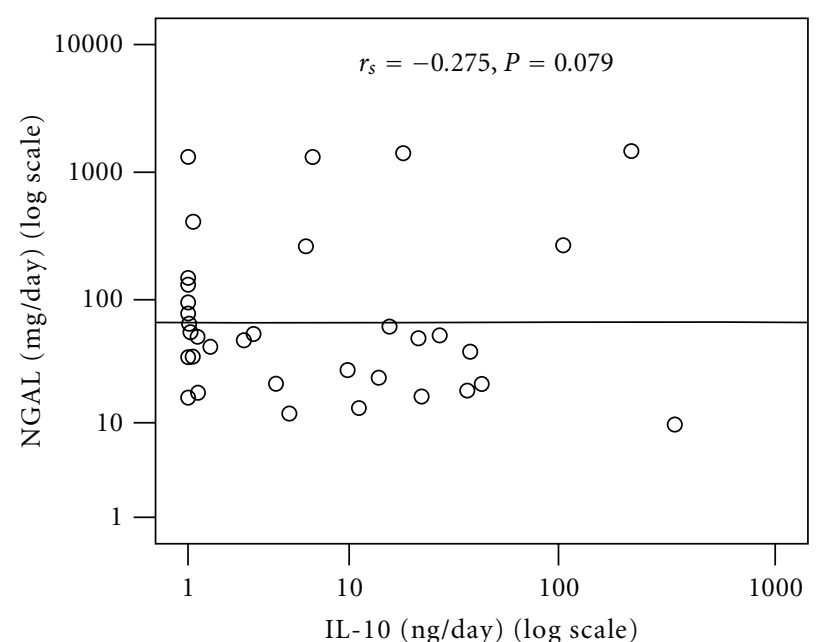

(a)

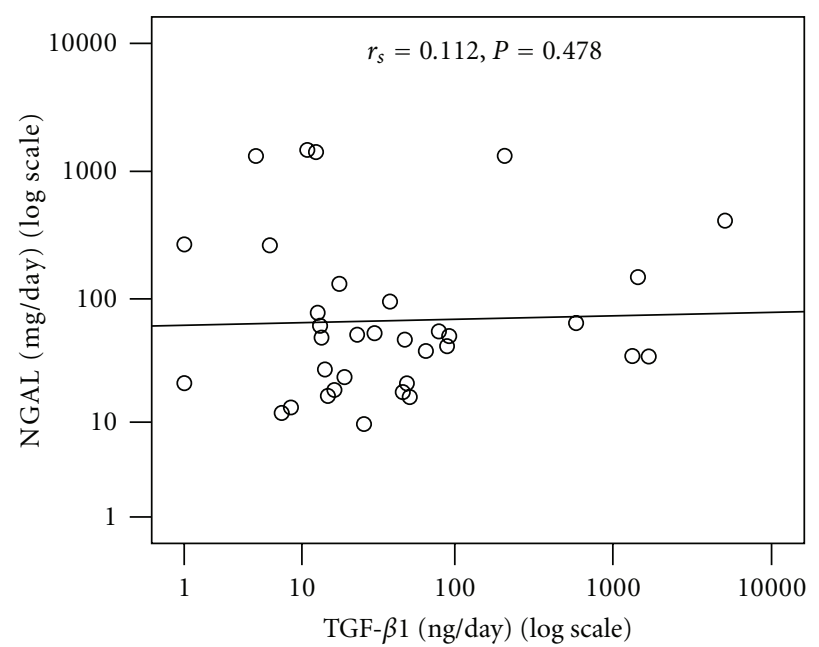

(c)

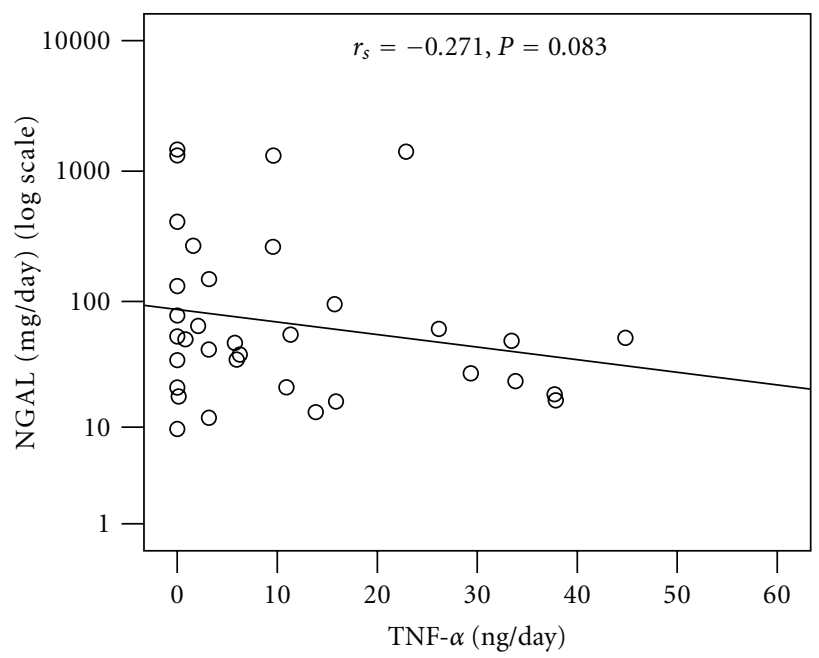

(e)

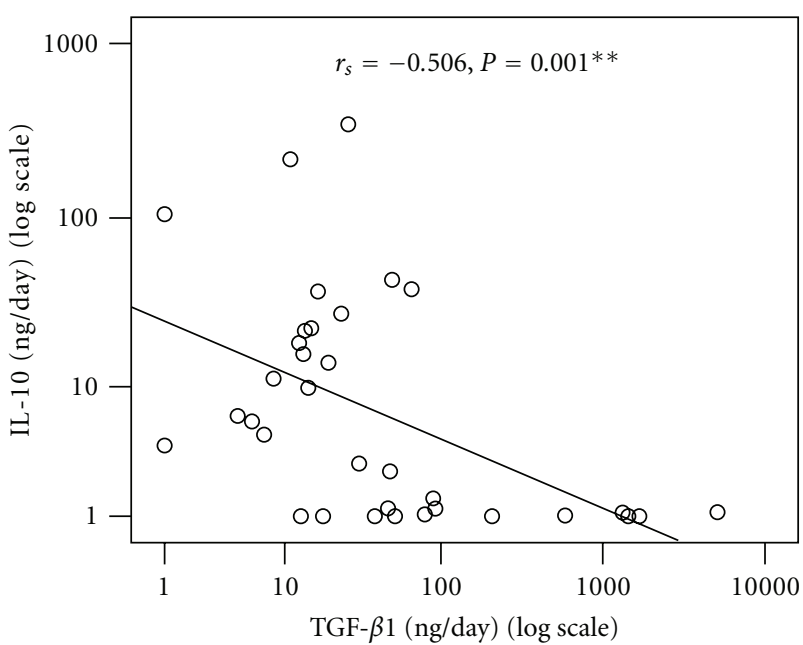

(b)

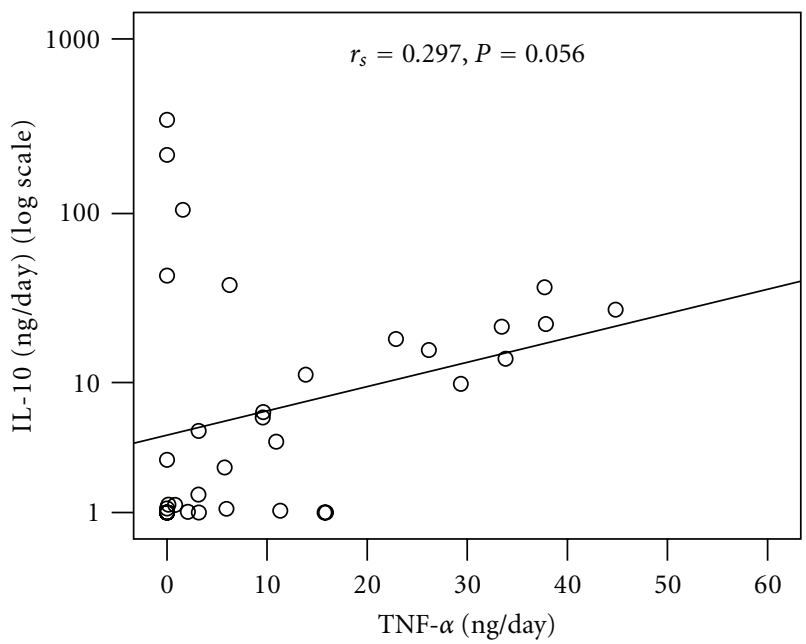

(d)

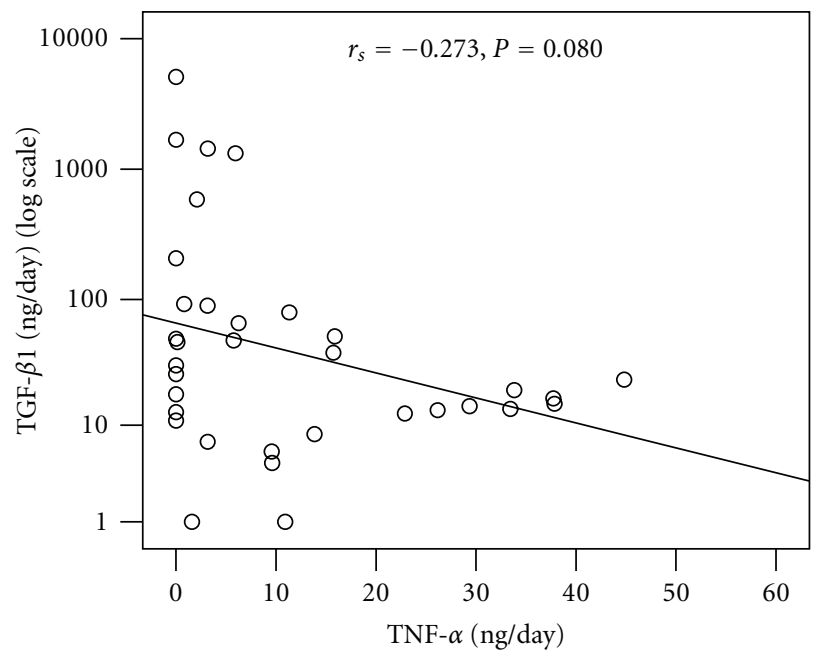

(f)

FIgURE 2: Correlations among 24-hour urinary excretion of neutrophil gelatinase-associated lipocalin (NGAL), interleukin (IL)-10, transforming growth factor (TGF)- $\beta 1$, tumor necrosis factor (TNF)- $\alpha$ in the systemic lupus erythematosus (SLE)-renal group. (a) Correlation between NGAL and IL-10. (b) Correlation between IL-10 and TGF- $\beta 1$. (c) Correlation between NGAL and TGF- $\beta 1$. (d) Correlation between IL-10 and TNF- $\alpha$ (e) Correlation between NGAL and TNF- $\alpha$ and (f) Correlation between TGF- $\beta 1$ and TNF- $\alpha$. $* * P<0.01$. 


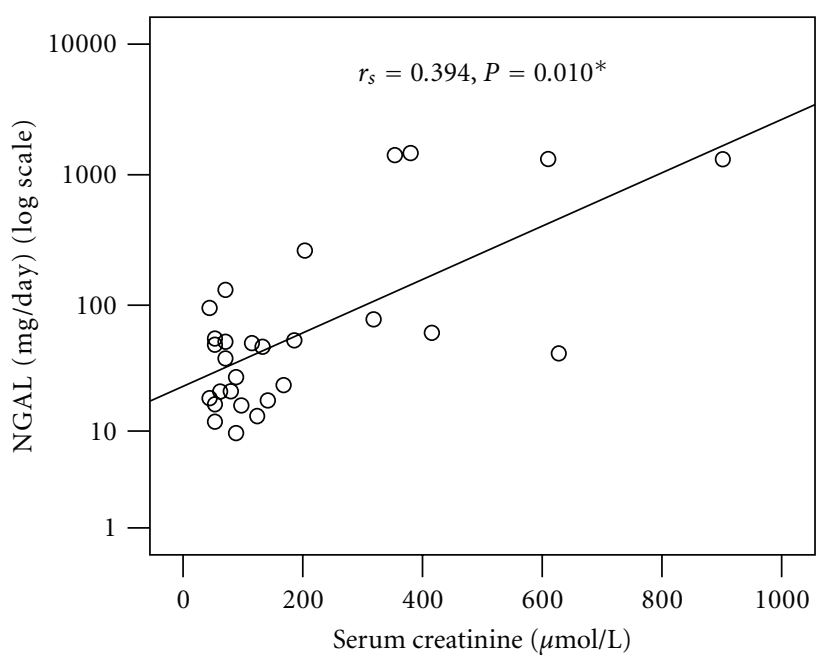

(a)

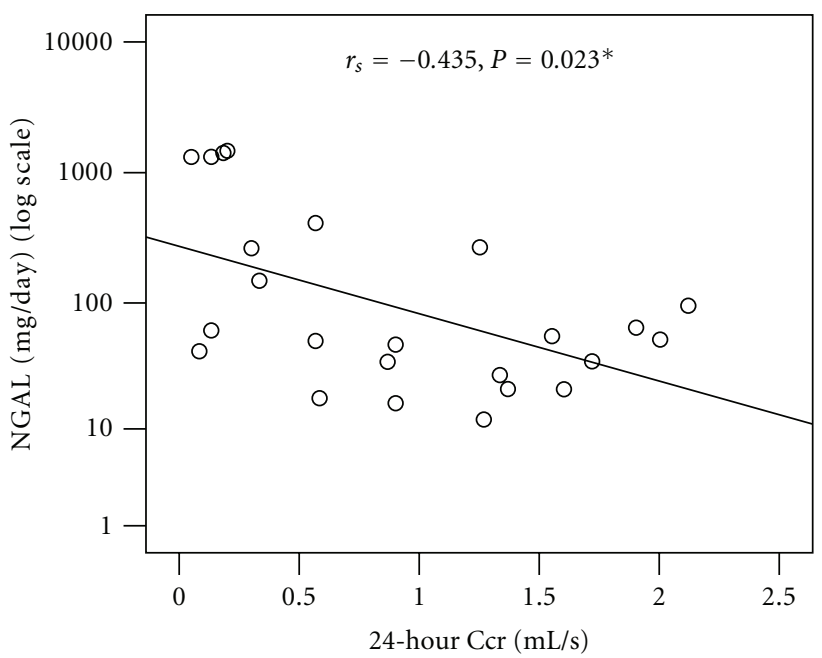

(b)

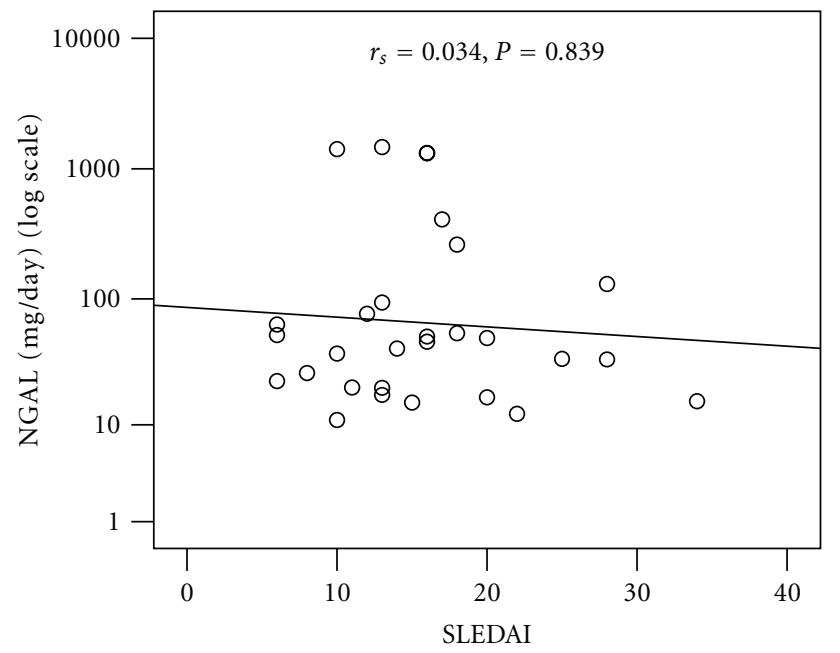

(c)

FIGURE 3: Correlations of 24-hour urinary excretion of neutrophil gelatinase-associated lipocalin (NGAL) with renal functional parameters and Systemic Lupus Erythematosus Disease Activity Index (SLEDAI) scores in the systemic lupus erythematosus (SLE)-renal group. (a) Correlation between NGAL and serum creatinine levels. (b) Correlation between NGAL and 24-hour creatinine clearance (Ccr). (c) Correlation between NGAL and SLEDAI scores. ${ }^{*} P<0.05$.

levels $\left(r_{s}=-0.263, P=0.110\right)$, 24-hour urine protein amounts $\left(r_{s}=0.288, P=0.068\right)$, C3 levels $\left(r_{s}=0.015, P=\right.$ $0.930)$, C4 levels $\left(r_{s}=0.292, P=0.093\right)$, and anti-dsDNA antibody titers $\left(r_{s}=-0.155, P=0.383\right)$ (data not shown). There was also no significant correlation with SLEDAI scores $\left(r_{s}=0.034, P=0.839\right.$; Figure $\left.3(\mathrm{c})\right)$. These results further confirm that the higher urinary NGAL excretion in the SLErenal group seems to derive from renal damage rather than from active immune reactions in the kidneys.

\subsection{Logistic Regression Analysis of 24-Hour Urinary NGAL} Excretion As a Reliable Predictor for Renal Damage in SLE Patients. Using a logistic regression univariate model with renal involvement as the outcome and 24-hour urinary NGAL excretion as a predictor, we found that urinary NGAL excretion was a significant predictor for the SLE-renal group in all study patients $(P=0.008$; odds ratio (OR), 1.060; 95\% confidence interval (CI), 1.015-1.107); however, the result was not significant when the study population was confined to the SLE patients $(P=0.099$; OR, $1.049 ; 95 \%$ CI, 0.991-1.110). After further adjustment for age and sex, urinary NGAL excretion remained a significant predictor for the SLE-renal group in all patients $(P=0.013$; OR, 1.060; 95\% CI, 1.012-1.111).

3.6. ROC Curve Analysis of 24-Hour Urinary NGAL Excretion to Identify SLE Patients with Renal Involvement. To quantify the diagnostic value of 24-hour urinary NGAL excretion in patients with renal involvement, we performed the ROC curve analysis. When the study population was confined to the SLE patients, the AUC for urinary NGAL excretion was $0.755(P=0.033$; 95\% CI, 0.587-0.924; Figure 4(a)). 


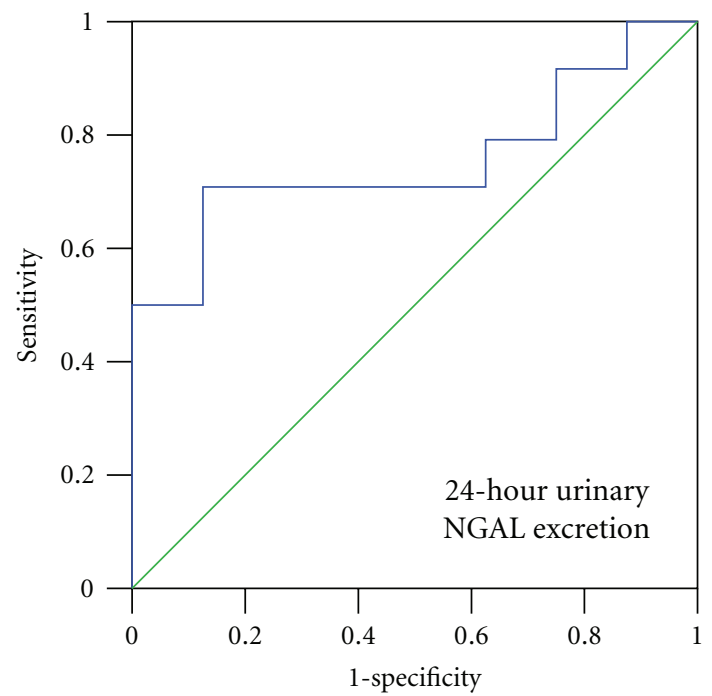

(a)



(b)

FIGURE 4: Detection of sensitivity and specificity of 24-hour urinary neutrophil gelatinase-associated lipocalin (NGAL) excretion (a) and antidouble stranded DNA (anti-dsDNA) titers (b) for the diagnosis of renal involvement in systemic lupus erythematosus (SLE) patients by receiver operating characteristic curve (ROC) analysis.

In comparison, the AUC for anti-dsDNA antibody titers was $0.600(P=0.416$; 95\% CI, 0.336-0.864; Figure 4(b)). Therefore, urinary NGAL excretion is a better biomarker than anti-dsDNA antibody titers for SLE patients with renal involvement in our study. Table 3 lists the calculated sensitivity, specificity, positive predictive values, and negative predictive values of 24-hour urinary NGAL excretion at different cut-off values for the diagnosis of renal involvement in SLE patients.

\section{Discussion}

Our study demonstrates that urinary NGAL, rather than IL-10, TGF- $\beta 1$, and TNF- $\alpha$, is a potential biomarker for renal damage in SLE patients. The evidence is supported by the following 3 facts: (1) Urinary NGAL excretion was higher in SLE patients with renal involvement than in the SLE-non-renal or non-SLE autoimmune disease groups. (2) Urinary NGAL excretion was positively correlated with serum creatinine levels and negatively correlated with 24hour Ccr in SLE patients with renal involvement. (3) Urinary NGAL excretion had higher sensitivity and specificity as well as a higher positive predictive value for the diagnosis of renal involvement in SLE patients than did anti-dsDNA antibody titers.

NGAL was originally identified as a protein associated with purified gelatinase from the supernatant of activated neutrophils [20]. Subsequent murine and human studies revealed remarkable expression levels of NGAL mRNA and protein in the tubular cells of early postischemic kidneys [21]. Accordingly, NGAL was regarded as a novel biomarker in various types of acute kidney injury and chronic kidney diseases. It is now conceivable that NGAL is an acute phase reactant rapidly released from various cell types including neutrophils, monocytes/macrophages, epithelial cells, vascular endothelial cells, and hepatocytes [16, 17].

Although the SLE-non-renal group exhibited relatively lower disease activity (median SLEDAI score, 8) than the SLE-renal group (median SLEDAI score, 14), we observed no correlation between urinary NGAL excretion and the SLEDAI score. Furthermore, no correlation was observed with IL-10, TGF- $\beta 1$, or TNF- $\alpha$ levels, which are regarded as markers of the active immune response in SLE. We postulate that urinary NGAL excretion is more closely related to renal damage than to systemic or intrarenal immune reactions. Our data is compatible with those of other authors who observed that higher urinary NGAL levels in LN were not necessarily correlated with SLEDAI scores in both pediatric [22] and adult SLE patients [23]. In contrast, urinary NGAL was correlated with worsening renal disease activity in pediatric SLE patients [22]. Rubinstein et al. [24] argued that NGAL was a significant predictor of renal disease activity and renal flares in SLE patients.

It is interesting to identify the source of NGAL in the urine of SLE patients with renal involvement. We believe that plasma NGAL is unrelated to urinary NGAL in SLE patients based on the observation that plasma NGAL did not differ significantly between SLE patients with or without active global or renal disease $[22,23]$. Although we did not measure plasma NGAL in our patients, we deduce that either enhanced local production from renal tubular cells or increased NGAL leakage from glomerular capillaries is the major source of increased urinary NGAL in the SLErenal group in this study. Bolignano et al. [16] proposed that the increase in urinary NGAL in chronic renal proteinuric diseases is due to massive protein loss that may saturate megalin-cubilin transporters on renal tubular cells leading to 
TABle 3: Sensitivity, specificity, positive predictive values, and negative predictive values of 24-hour urinary NGAL excretion at different cut-off values for the diagnosis of renal involvement in SLE patients.

\begin{tabular}{lcccc}
\hline Cutoff value $(\mathrm{mg} /$ day) & Sensitivity $(\%)$ & Specificity $(\%)$ & Positive predictive value $(\%)$ & Negative predictive value (\%) \\
\hline 24.00 & 70.8 & 62.5 & 85 & 41.7 \\
27.00 & 70.8 & 75 & 84.5 & 46.2 \\
31.00 & 70.8 & 87.5 & 94.4 & 50 \\
36.00 & 66.7 & 87.5 & 94.1 & 46.7 \\
\hline
\end{tabular}

NGAL: neutrophil gelatinase-associated lipocalin; SLE: systemic lupus erythematosus.

reduced NGAL reabsorption. Consequently, the intratubular complement cascade activation by persistent proteinuria would further damage tubular cells that exhibit impaired reabsorption via megalin-cubilin transporters. In addition to passive NGAL leakage into the urine, the injured tubular cells may actively produce NGAL as a compensatory mechanism against complement-induced oxidative stress and apoptosis. These findings suggest that renal damage with or without inflammation may enhance NGAL excretion, as shown in our SLE-renal group.

NGAL is normally expressed at very low levels in human renal tubular cells [25]; this is also seen in our study. NGAL mRNA and protein expression is rapidly induced in the proximal renal tubular cells after toxic or ischemic kidney injury $[21,26]$. In diabetic mice, increased urinary NGAL was mainly due to impaired renal reabsorption. In mice with obstructive nephropathy, NGAL protein was rapidly synthesized in the distal nephrons in the obstructed side of the kidneys and in the proximal renal tubules in the contralateral side [27]. The LCN2 gene, which encoded the NGAL protein, was markedly expressed in the glomerular mesangial cells in the presence of nephritogenic murine anti-dsDNA monoclonal antibodies in vitro and in vivo [28]. Upregulated glomerular expression of the LCN2 gene and increased urinary NGAL excretion were also found in crescentic glomerulonephritis in murine chronic graftversus-host-disease (a model for human SLE) [29]. In a human study on IgA nephropathy, strong staining for the NGAL protein was found only in the proximal tubules and not in the glomeruli or tubulointerstitial space in patients with Lee grade III disease [25].

Cell types other than renal tubular cells in the body, such as various types of epithelial cells, endothelial cells, or immune cells, might contribute to urinary NGAL levels in patients even without nephritis. It is not surprising that those patients without renal involvement, both in the SLE-nonrenal and non-SLE autoimmune disease groups, exhibited higher urinary NGAL excretion than did normal controls.

The role of NGAL in the pathogenesis of renal damage remains to be elucidated. In a murine model of ischemic kidney injury, administration of recombinant NGAL before ischemia or within 1 hour after reperfusion significantly decreased serum creatinine levels and apoptotic cell numbers, but increased the numbers of proliferating tubules cells [30]. In addition, NGAL also exerted an antiapoptotic effect, preserved $\mathrm{N}$-cadherin expression in proximal renal tubules, and upregulated the antioxidative enzyme heme oxygenase1 in ischemic murine kidneys [31]. Putting these together, NGAL may play a protective role in the repair and regeneration of injured renal tubular cells. In the present study, we also investigated the effects of medications including nephrotoxic agents (such as cyclophosphamide, aspirin, or nonsteroidal anti-inflammatory drugs) or nephroprotective agents (such as angiotensin-converting enzyme inhibitors or angiotensin II receptor antagonists) on urinary NGAL excretion, with inconclusive results.

Drawbacks of the present study include the following. (1) The patient numbers were relatively small. (2) Rather than early morning spot urine, we collected 24-hour urine, which was hard to maintain in a sterile condition; this might affect the accuracy of NGAL determination. (3) All SLE and non-SLE patients expressed diverse clinical manifestations, and disease activity of SLE patients was uniformly assessed by SLEDAI; this might complicate the statistical analysis. (4) We used stringent selection criteria for active nephritis, including the presence of cellular urine sediments, a $>30 \%$ increase in serum creatinine levels within 3 months, or biopsy-proven nephritis, which reflected active renal inflammation. In consideration of possible irreversible damage to the glomerular basement membrane and lack of active renal inflammation, we classified a group of SLE patients with only proteinuria ( $>0.5 \mathrm{~g} /$ day) as the SLE-renal group along with those that were included in the SLE-active nephritis group. However, the diagnosis of active nephritis in our study was primarily based on laboratory data and rarely on renal pathological findings. Therefore, we could not analyze the relationship of urinary NGAL excretion with histopathological changes in active nephritis. (5) We used the commonly used SLEDAI scoring system to assess the disease activity of the SLE patients. It has the advantage of facilitating global evaluation of the patients, but lacks a severity grading that reduces its sensitivity $[32,33]$. (6) It was difficult to analyze the effects of diverse medications on the excretion of urinary inflammatory markers.

\section{Conclusion}

Our study demonstrated that urinary NGAL is a potential biomarker for renal damage in SLE patients. Direct demonstration of NGAL mRNA and protein expression in the lupus kidney by in situ hybridization, and immunohistochemical 
staining studies, are needed to clarify the origin of NGAL in SLE patients.

\section{Conflict of Interests}

The authors declare that they have no conflict of interests.

\section{Acknowledgments}

This study was supported by a grant from the National Sciences Council (NSC99-2628-B-002-020-MY3). The authors are indebted to the Clinical Immunology and Transplantation Research Center, the Eighth Core Lab, Department of Medical Research, and National Taiwan University Hospital for providing space and laboratory facilities.

\section{References}

[1] N. Schwartz, J. S. Michaelson, and C. Putterman, "Lipocalin-2, TWEAK, and other cytokines as urinary biomarkers for lupus nephritis," Annals of the New York Academy of Sciences, vol. 1109, pp. 265-274, 2007.

[2] J. E. Balow, "Clinical presentation and monitoring of lupus nephritis," Lupus, vol. 14, no. 1, pp. 25-30, 2005.

[3] C. C. Mok, "Prognostic factors in lupus nephritis," Lupus, vol. 14, no. 1, pp. 39-44, 2005.

[4] P. I. Sidiropoulos, H. D. Kritikos, and D. T. Boumpas, "Lupus nephritis flares," Lupus, vol. 14, no. 1, pp. 49-52, 2005.

[5] C. Fiehn, "Early diagnosis and treatment in lupus nephritis: how we can influence the risk for terminal renal failure," The Journal of Rheumatology, vol. 33, no. 8, pp. 1464-1466, 2006.

[6] P. Enghard and G. Riemekasten, "Immunology and the diagnosis of lupus nephritis," Lupus, vol. 18, no. 4, pp. 287290, 2009.

[7] R. W. Y. Chan, F. M. M. Lai, E. K. M. Li et al., "Expression of chemokine and fibrosing factor messenger RNA in the urinary sediment of patients with lupus nephritis," Arthritis and Rheumatism, vol. 50, no. 9, pp. 2882-2890, 2004.

[8] Y. Avihingsanon, P. Phumesin, T. Benjachat et al., "Measurement of urinary chemokine and growth factor messenger RNAs: a noninvasive monitoring in lupus nephritis," Kidney International, vol. 69, no. 4, pp. 747-753, 2006.

[9] A. M. Hammad, H. M. Youssef, and M. M. El-Arman, "Transforming growth factor beta 1 in children with systemic lupus erythematosus: a possible relation with clinical presentation of lupus nephritis," Lupus, vol. 15, no. 9, pp. 608-612, 2006.

[10] R. W. Y. Chan, L. S. Tam, E. K. M. Li et al., "Inflammatory cytokine gene expression in the urinary sediment of patients with lupus nephritis," Arthritis and Rheumatism, vol. 48, no. 5, pp. 1326-1331, 2003.

[11] B. H. Rovin, H. Song, D. J. Birmingham, L. A. Hebert, C. Y. $\mathrm{Yu}$, and H. N. Nagaraja, "Urine chemokines as biomarkers of human systemic lupus erythematosus activity," Journal of the American Society of Nephrology, vol. 16, no. 2, pp. 467-473, 2005.

[12] N. Schwartz, L. Su, L. C. Burkly et al., "Urinary TWEAK and the activity of lupus nephritis," Journal of Autoimmunity, vol. 27, no. 4, pp. 242-250, 2006.

[13] N. Schwartz, T. Rubinstein, L. C. Burkly et al., "Urinary TWEAK as a biomarker of lupus nephritis: a multicenter cohort study," Arthritis Research \& Therapy, vol. 11, no. 5, article R143, 2009.
[14] T. Wu, C. Xie, H. W. Wang et al., "Elevated urinary VCAM1, P-selectin, soluble TNF receptor-1, and CXC chemokine ligand 16 in multiple murine lupus strains and human lupus nephritis," Journal of Immunology, vol. 179, no. 10, pp. 71667175, 2007.

[15] D. H. Goetz, M. A. Holmes, N. Borregaard, M. E. Bluhm, K. N. Raymond, and R. K. Strong, "The neutrophil lipocalin NGAL is a bacteriostatic agent that interferes with siderophoremediated iron acquisition," Molecular Cell, vol. 10, no. 5, pp. 1033-1043, 2002.

[16] D. Bolignano, V. Donato, G. Coppolino et al., "Neutrophil gelatinase-associated lipocalin (NGAL) as a marker of kidney damage," American Journal of Kidney Diseases, vol. 52, no. 3, pp. 595-605, 2008.

[17] T. Rubinstein, M. Pitashny, and C. Putterman, "The novel role of neutrophil gelatinase-B associated lipocalin (NGAL)/ Lipocalin-2 as a biomarker for lupus nephritis," Autoimmunity Reviews, vol. 7, no. 3, pp. 229-234, 2008.

[18] M. C. Hochberg, "Updating the American College of Rheumatology revised criteria for the classification of systemic lupus erythematosus," Arthritis and rheumatism, vol. 40, no. 9, article 1725, 1997.

[19] D. D. Gladman, D. Ibañez, and M. B. Urowltz, "Systemic lupus erythematosus disease activity index 2000," The Journal of Rheumatology, vol. 29, no. 2, pp. 288-291, 2002.

[20] L. Kjeldsen, A. H. Johnsen, H. Sengelov, and N. Borregaard, "Isolation and primary structure of NGAL, a novel protein associated with human neutrophil gelatinase," The Journal of Biological Chemistry, vol. 268, no. 14, pp. 10425-10432, 1993.

[21] J. Mishra, M. A. Qing, A. Prada et al., "Identification of neutrophil gelatinase-associated lipocalin as a novel early urinary biomarker for ischemic renal injury," Journal of the American Society of Nephrology, vol. 14, no. 10, pp. 2534-2543, 2003.

[22] M. Suzuki, K. M. Wiers, M. S. Klein-Gitelman et al., "Neutrophil gelatinase-associated lipocalin as a biomarker of disease activity in pediatric lupus nephritis," Pediatric Nephrology, vol. 23, no. 3, pp. 403-412, 2008.

[23] M. Pitashny, N. Schwartz, X. Qing et al., "Urinary lipocalin2 is associated with renal disease activity in human lupus nephritis," Arthritis and Rheumatism, vol. 56, no. 6, pp. 18941903, 2007.

[24] T. Rubinstein, M. Pitashny, B. Levine et al., "Urinary neutrophil gelatinase-associated lipocalin as a novel biomarker for disease activity in lupus nephritis," Rheumatology, vol. 49, no. 5, pp. 960-971, 2010.

[25] H. Ding, Y. He, K. Li et al., "Urinary neutrophil gelatinaseassociated lipocalin (NGAL) is an early biomarker for renal tubulointerstitial injury in IgA nephropathy," Clinical Immunology, vol. 123, no. 2, pp. 227-234, 2007.

[26] J. Mishra, K. Mori, Q. Ma, C. Kelly, J. Barasch, and P. Devarajan, "Neutrophil gelatinase-associated lipocalin: a novel early urinary biomarker for cisplatin nephrotoxicity," American Journal of Nephrology, vol. 24, no. 3, pp. 307-315, 2004.

[27] T. Kuwabara, K. Mori, M. Mukoyama et al., "Urinary neutrophil gelatinase-associated lipocalin levels reflect damage to glomeruli, proximal tubules, and distal nephrons," Kidney International, vol. 75, no. 3, pp. 285-294, 2009.

[28] X. Qing, J. Zavadil, M. B. Crosby et al., "Nephritogenic antiDNA antibodies regulate gene expression in MRL/lpr mouse glomerular mesangial cells," Arthritis and Rheumatism, vol. 54, no. 7, pp. 2198-2210, 2006.

[29] S. M. Ka, A. Rifai, J. H. Chen et al., "Glomerular crescentrelated biomarkers in a murine model of chronic graft versus 
host disease," Nephrology Dialysis Transplantation, vol. 21, no. 2, pp. 288-298, 2006.

[30] J. Mishra, K. Mori, Q. Ma et al., "Amelioration of ischemic acute renal injury by neutrophil gelatinase-associated lipocalin," Journal of the American Society of Nephrology, vol. 15, no. 12, pp. 3073-3082, 2004.

[31] K. Mori, H. T. Lee, D. Rapoport et al., "Endocytic delivery of lipocalin-siderophore-iron complex rescues the kidney from ischemia-reperfusion injury," The Journal of Clinical Investigation, vol. 115, no. 3, pp. 610-621, 2005.

[32] M. M. Ward, A. S. Marx, and N. N. Barry, "Comparison of the validity and sensitivity to change of 5 activity indices in systemic lupus erythematosus," The Journal of Rheumatology, vol. 27, no. 3, pp. 664-670, 2000.

[33] L. A. Mandl and M. H. Liang, "Monitoring patients with systemic lupus erythematosus," in Systemic Lupus Erythematosus, R. G. Lahita, Ed., p. 626, Elsevier Academic Press, Amsterdam, The Netherlands, 4th edition, 2004. 


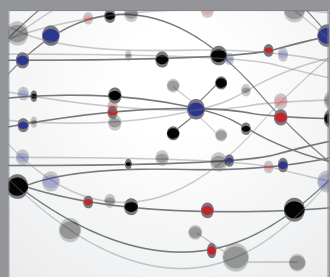

The Scientific World Journal
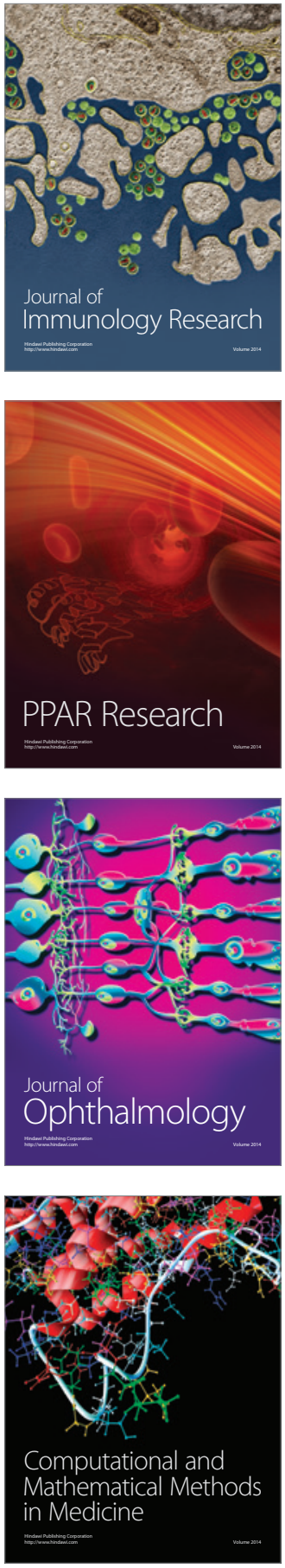

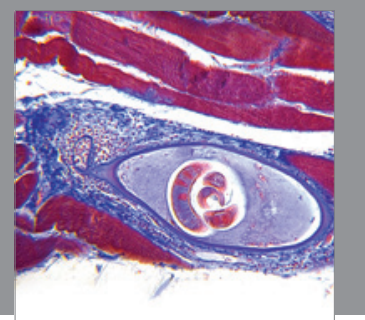

Gastroenterology

Research and Practice
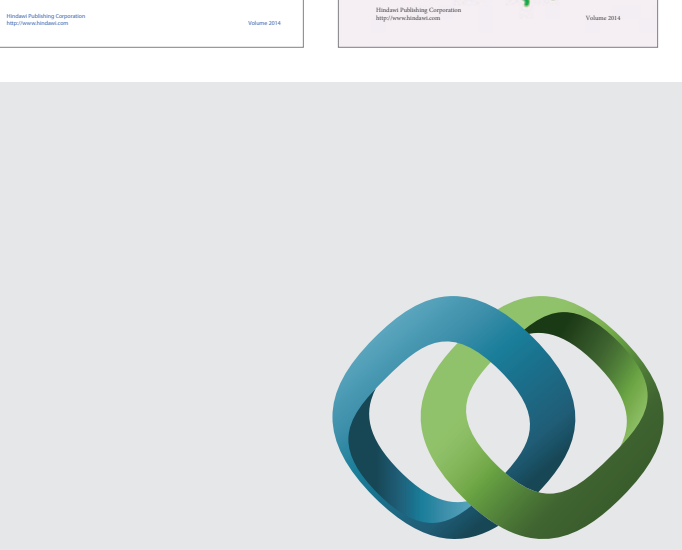

\section{Hindawi}

Submit your manuscripts at

http://www.hindawi.com
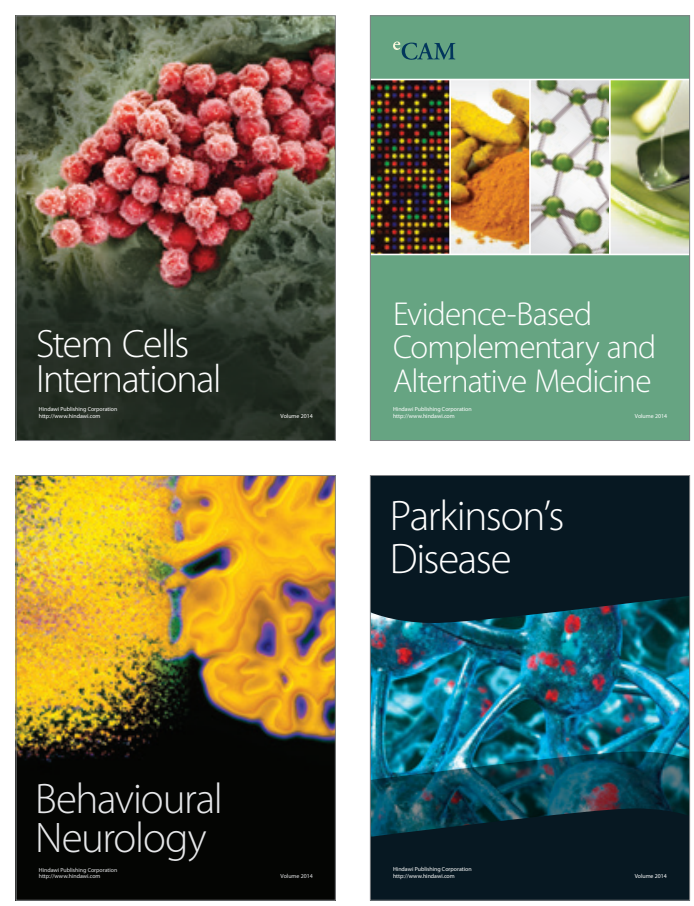



Journal of
Diabetes Research

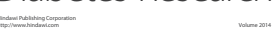

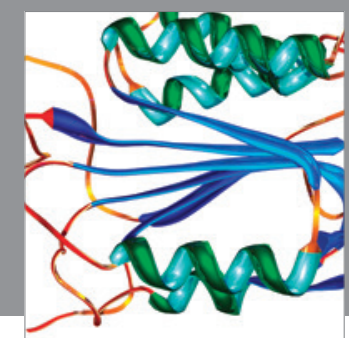

Disease Markers
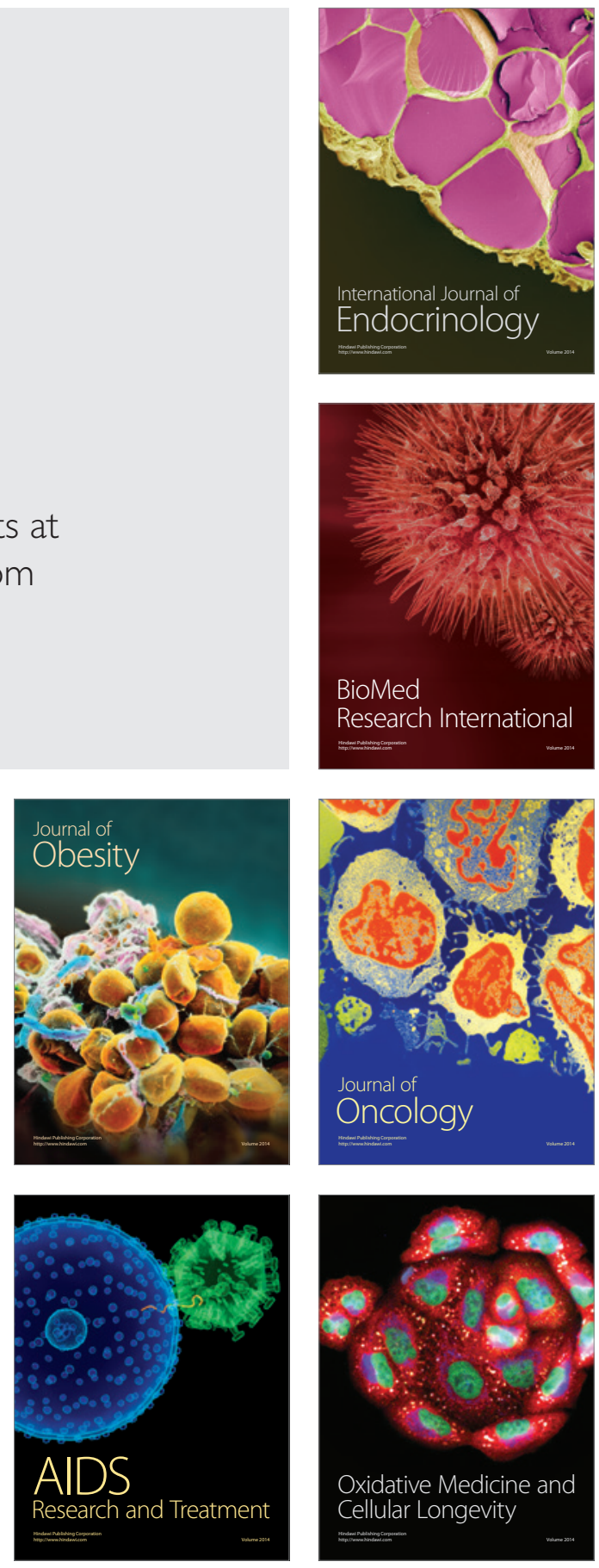